\title{
A review of cloud oriented mobile learning platform and frameworks
}

\author{
Ahmad Shukri Bin Moh Noor, Marwan Nasser Yousef Atoom, Rabiei Mamat \\ School of Informatics and Applied Mathematics, Universiti Malaysia Terengganu, Malaysia
}

\begin{tabular}{l}
\hline Article Info \\
\hline Article history: \\
Received Nov 30, 2018 \\
Revised Apr 18, 2019 \\
Accepted Jun 20, 2019 \\
\hline
\end{tabular}

\section{Keywords:}

Cloud based mobile learning

Cloud computing

Framework

Mobile device

Mobile learning

\begin{abstract}
With the continued growth of mobile devices usage, wireless communications improvement, and cloud computing evolution, many educational institutions around the world, especially universities and colleges, began to provide their students with mobile learning systems based on cloud computing. The widespread, ubiquitous, and flexible natures of mobile devices make mobile learning an attractive alternative in education, particularly when integrating it with cloud computing which is the up-to-date technology that delivers computing hardware and software as services. However, the participatory between mobile learning and cloud computing as a cloud based mobile learning (CBML) becomes one of the important methods in the learning process. Many researches have attempted to combine the unique features of CBML in a form of frameworks. These frameworks have been designed to identify, categorize, or evaluate the major components of the CBML system. This paper is an attempt to identify the important role of cloud computing technology in mobile learning, investigate the main advantages and limitations of CBML systems, and explore the previously designed CBML frameworks.
\end{abstract}

Copyright $@ 2019$ Institute of Advanced Engineering and Science. All rights reserved.

\section{Corresponding Author:}

Ahmad Shukri Bin Moh Noor,

School of Informatics and Applied Mathematic,

Universiti Malaysia Terengganu,

Terengganu, Malaysia.

Email: ashukri@umt.edu.my

\section{INTRODUCTION}

The concept of mobile learning has become common and familiar in education as a result of increasing usage of mobile devices which are the most convenient and effective communication tools in our daily life. It is considered as a future learning model [1] and as a type of learning that takes facilities and advantages from mobile devices and its applications where learners are not at a predetermined and fixed location [2] in order to support the learning and teaching process anywhere and anytime. Hopefully, mobile learning will improve and develop the learning process in general and lead to smart educational systems.

In the last few years, storing documents online is a fanciful idea. Nowadays, data transferred from terminal devices and stored at servers on the cloud over the Internet. This technology helps in storing data outside computer context and becomes part of our daily life. Cloud computing is a compelling paradigm for delivering and managing services over the Internet [3]. It is a model for enabling ubiquitous and on-demand network access to a shared pool of configurable computing resources, like: servers, storage, networks, applications, and services which can be rapidly released and provisioned with a minimal effort [4]. In this regard, many cloud computing providers such as Microsoft, Google, IBM, and CISCO have provided smart solutions for education.

Mobile learning is getting widespread day by day in amalgamation with cloud computing [5]. CBML is a new concept; it is an integration of mobile learning and cloud computing [6] that aims to 
overcome obstacles related to mobile computing [7]. It refers to a structure where learning materials as well as data storage and execution of learners and educational institutions are not at mobile devices; instead, they move a way to the cloud [8] which promises for a great development of the educational in the future [9]. So, many educational institutions, especially universities, have recognized the benefits and potentials of using CBML system [10].

CBML has become a big issue in many researches, mainly at higher educational institutions (HEIs). Based on the expected outcomes of CBML systems, many researchers have made efforts to explore the effects of such systems at education [11]. Others have proposed CBML frameworks derived from different perspectives in order to identify, investigate, explore, categorize, evaluate the pedagogical features of CBML systems [12, 13].

A framework is a comprehensive overview, a conceptual or real structure, proposed to serve as a guide or support for building things. It is a particular set of ideas, beliefs, or rules which can be used to decide what to do or to deal with problems. The main goal of CBML frameworks is getting benefits from the wide spread of mobile devices technologies and the possibilities of cloud computing tools in order to introduce mobile learning solutions [14] that promise in making the learning process easier, flexible, effective, and ubiquitous.

\section{OBJECTIVES}

The main objectives of this study are to introduce and investigate the existing CBML frameworks in order to provide an essential knowledge and resources to improve the quality of CBML systems which leads to the development of the learning process, shed light on the importance of cloud computing technology in supporting mobile learning systems, and discuss the advantages and limitations of CBML systems.

\section{SUPPORTING MOBILE LEARNING WITH CLOUD COMPUTING}

When designing a mobile learning system, technical issues are very essential to be considered, involves: how to implement and manage the learning material and learners' preferences and profiles? What technologies should be used to provide support to different mobile devices? Also, there is a nontechnical issue of how to improve the learning experiences and learners' performance (learning outcomes)?

There are many challenges that face the implementation of mobile learning. Some of these challenges are related to the using of mobile devices. So, in order to make the mobile learning system more effective, there is a great need to take the facilities from other new technologies. Some of challenges in mobile devices are the small storage capacity and the limited processing power. Cloud computing eliminates these challenges by offering a gigantic storage capacity and a very speed processing power for mobile learning. Cloud computing gives mobile learners more flexibility in completing assignments, sharing files, accessing all documents types, etc., as it is available in more sophisticated computer devices. Cloud computing is the storing, processing, and managing of data, this is by using a remote server hosted on the Internet in preference to a personal computer or a local server. So, researchers hold a great hope in integrating mobile learning with cloud computing [15] as it is the best solution for mobile learning systems with dynamic scalability and low cost [16].

Utilizing cloud computing in learning is ideal as it permits reaching of educational resources to a huge number of learners. The integration of cloud computing and mobile learning promotes mobile learning system to have a rich content, more powerful, and more widely applied. Therefore, there is a strong interest relation between mobile learning and cloud computing, this interest can't be separated since mobile learning can benefit tremendously from cloud computing environment. With some investment in mobile learning infrastructure, even small educational institutions can initiate in taking the facilities of the cloud. So, the developers of mobile learning applications have adopted the cloud computing technology services as a storage tool and a universal computation resource. Accordingly, mobile learning efficiency could be achieved by integrating it with cloud computing.

\section{ADVANTAGES OF CBML}

There are many advantages of CBML systems. It is worthy to say that, with mobile devices and cloud computing, learning becomes portable. As mobile devices are portable and the cloud computing is available anywhere and anytime, CBML gives learners a wide range of flexibility in space and time. So, it removes the spatial and temporal limitations. Also, the availability of learning contents, learners' data, information, references, learning activities, time management tools, and assessments at mobile devices helps in removing barriers of learning process and support synchronized content. 
In terms of cost, CBML cuts down the cost of data storage and processing in educational institutions; the low cost of mobile devices and cloud services is the most main benefit of CBML in comparing with the desktop computers or laptops. Also, educational institutions do not need to upgrade and maintain its software or hardware because all storage, updates, and computing processes will be completed on the cloud side; the function of the mobile device is displaying the learning contents only.

CBML is flexible; it supports the flexibility of the learning process with more varied course content formats; like: texts, images, videos, and audios. Also, it supports multi-devices; the same course is available on various mobile devices, ranging from tablets, smart phones, APDs, etc. Hence, it offers promising methods for learners to choose, handle, and apply information to their own needs in their learning environment which support and encourage personalized learning. On the other hand, using massive cloud computing infrastructure with mobile learning is possible to develop, test, and deliver new educational tools in order to transform the experiences of learning to meet the learners' needs which are frequently in changing.

CBML reforms the formal education; learners focus on getting knowledge, it brings the classroom to the learners. So, learners can download the content by just clicking on a screen. Similarly, it reforms assignments and assessments in a more comprehensive and easy way; assignments could be done in groups while each student is far away from the others. Besides that, learning resources are stored in the cloud. So, it can be shared among different educational institutions. Also, services which improve the learning process from different institutions can be shared. Therefore, more educational resources are available for learners.

CBML makes learners proactive in identifying their needs and in finding suitable solutions. It enhances communication, supports collaborative learning, and provides conversation space between learners themselves and with their instructors. It reduces the cultural and communication barriers between learners and instructors by using suitable channels that learners like. Also, learners can enjoy a high degree of collaboration by connecting them with teachers and experts. On the other hand, CBML may be used as a mean to engage disinterested learners to be involved in the learning process. In general, a new technology in any field usually motivates users.

One of the most benefits of CBML is the ability of storing gigantic data; it will provide educational institutions almost unlimited storage capacities. Mobile devices have limited storage capacities; CBML overcomes obstacles related to data storage. Also, mobile devices have limited computational power. Cloud computing supports unlimited computational power and high performance computing.

The good CBML system has the ability to analyze the learners' needs. It provides a high quality educational process, improves the quality of learning, and speeds the feedback to learners. Also, evaluating the effectiveness of the learning process becomes easier. CBML is easy to be controlled and monitored which helps in evaluating the learning process and its outcomes.

Mobile learning itself is an interactive and fun method that motivates learners; it makes the learning process more interesting, attractive, and enjoyable. Also, it gets rid of carrying heavy books and bags, everything students need is a very light mobile device connected to the cloud.

In general, CBML makes educational institutions focus on the core of education; the role of the cloud is to assist educational institutions to focus on their business. In addition, it is a prestige for educational institutions that uses mobile devices and cloud computing in its learning process; building an image as a modern educational institution.

\section{LIMITATIONS OF CBML}

Despite of all previous advantages of applying CBML systems, there are some challenges that restrict and limit the using of it. In this regard, privacy, security and safety are the biggest issues in CBML; every component in the cloud is accessible from the Internet. Naturally, nothing connected to the Internet is perfectly secure from attacking. So, privacy, security and safety are still the main obstacles in adopting CBML systems, especially in managing sensitive data of learners and educational institutions. Huge amount of data and software in multiple channels can be hacked easily through networks. So, educational institutions' data and learners' personal information could be misused.

In CBML, learners present negative issues related to mobile devices, which include: limited storage capacity, slow downloading, low access speed to online materials, limited batteries, low resolutions, poor colors, low contrast ratio, limited processing power, networks availability, and downtime. Normally, mobile devices' networks quality do not meet the learners' satisfactory. Furthermore, no cloud computing provider, even the best, has the power to provide its services during the Internet outages. CBML is Internet based, which means users' access is fully dependent on their Internet connection. On the other hand, there are many barriers in applying CBML related to the usability of mobile devices, like: small keyboards, small screen 
sizes, and the inability of editing documents. Also, in this regard, the digital environment is quite suitable for teenagers and children, but it requires efforts to be adopted and understood by adults.

The cost of unlimited Internet access by mobile devices and the prices of mobile devices are also barriers for some learners. This problem results from the rapid changes in mobile devices technologies; in models, functionality, capabilities, etc. Besides that, there are differences between cloud computing vendors systems in platforms, which make it difficult and may be impossible and expensive to migrate from one cloud computing platform to another.

Educational institutions that depend on CBML have limited control and management at their data, services, and applications. Also, there are inadequate regulations related to cloud computing technologies. In addition, like any software and hardware, CBML platforms may fail for any reasons and not all places over the globe are covered with wireless networks. So, the learning process may be interrupted at any time.

Evaluation challenges and assessment dilemma are also big issues, there is no crucial evidence regarding the effectiveness of adopting CBML in education. Learners' assessment still not clear in mobile learning and it is difficult to be applied outside the classroom. Moreover, with respect to learners' distraction; learners may spend their time in things that are not related and their attention may be easily unfocused during the educational process. In addition, ethical problems may appear publicly; ensuring of the proper use of mobile devices for educational purposes is a big challenge in CBML systems. Regarding health risks; people still suspect that using mobile devices over the time and at a long-term is one of the causes in increasing incidences of brain tumors, brain diseases, eyes problems, and aural problems which limit CBML usage.

\section{CBML FRAMEWORKS}

Generally, the use and development of CBML frameworks will confirm the effective employment of CBML systems in education and support the developmental process for transforming education to the Internet generation. Many researches have attempted to combine the unique features of CBML in a form of a framework. They attempted to identify, explore, categorize, or evaluate the components of the system. Some of these frameworks are presented in this section.

Brhanu and Mulugeta [17] designed a conceptual framework to adopt CBML for Ethiopian HEIs, as shown in Figure 1. Their efforts aim at investigating the difficulties facing cloud computing implementation in mobile learning. They took into their account technological factors and variety of strategic issues from areas of knowledge to ensure a successful CBML adoption, use the resources more effectively, and support the Quality of Service (QoS) objectives, such as availability, reliability, performance, scalability, load balancing, and security in the service models of the cloud. The framework consists of four horizontal layers: User Interface, SaaS, PaaS, and IaaS. Also, it comprises two vertical layers: Security Layer and Management and Integration layer. The researchers emphasized that their framework helps HEIs in adopting CBML successfully and will be the base for the development of CBML systems in an effective way. The researchers also proposed architecture for delivering mobile learning services through cloud. Their proposed architecture is shown in Figure 2, it consists of four layers: Users Layer, Devices Layer, Connection Layer, and Cloud Services Layer.

Alghabban et al. [18] developed a mobile learning framework architecture based on cloud computing. Their framework helps teachers and experts in teaching and assessing dyslexic students. They cited that the most considerable feature of their framework is that it fits all students' profiles and their preferences; this is by implementing learning profile content in different formats (texts, images, and audio). Their framework allows students to interact with the learning content through pointing on the mobile device screen. The proposed framework architecture consists of two parts connected together via the Internet, one part in the mobile device and the other in the cloud provider, as shown in Figure 3.

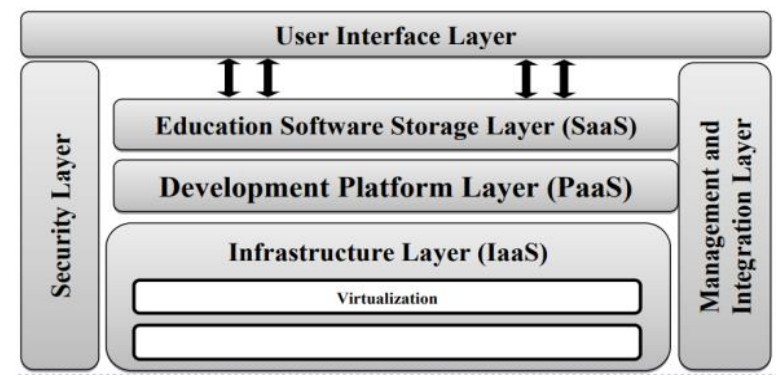

Figure 1. Conceptual framework for CBML [17] 


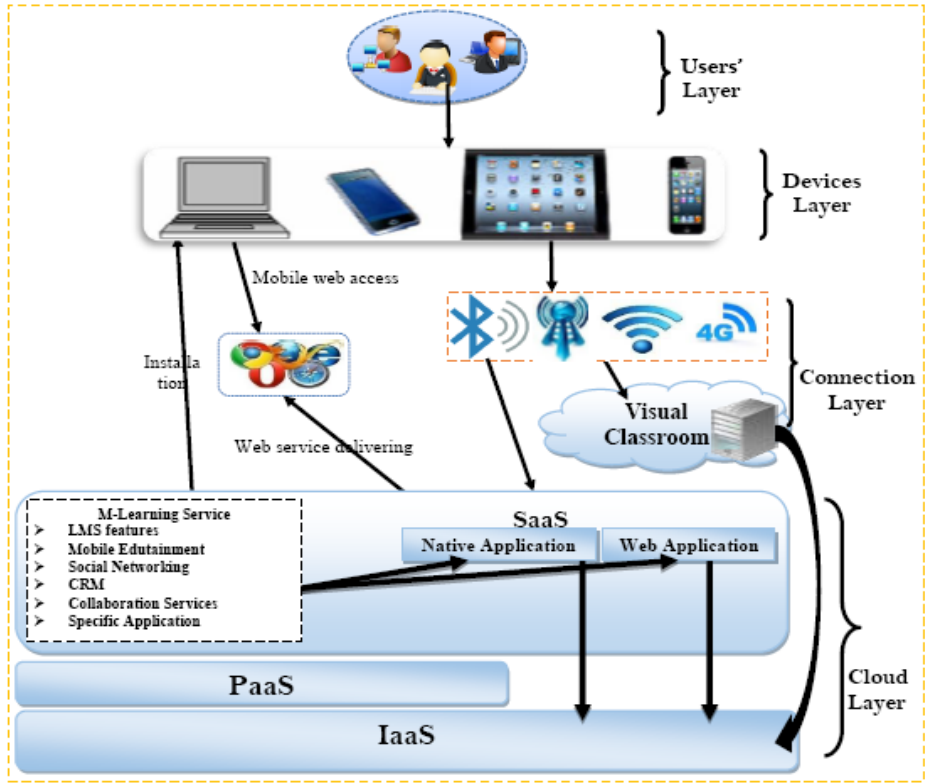

Figure 2. Architecture for delivering mobile learning services through cloud computing [17]

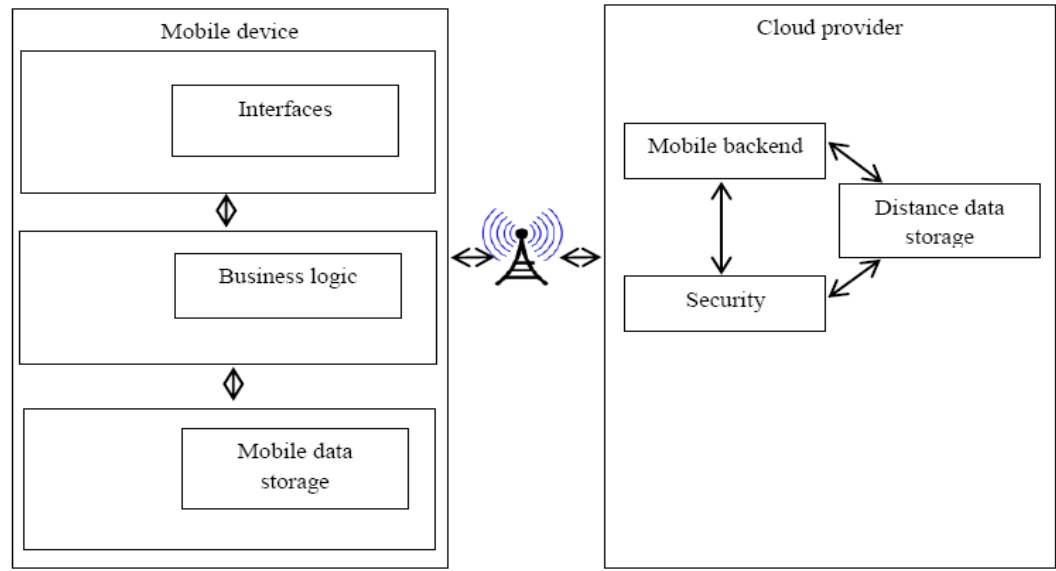

Figure 3. Mobile learning framework architecture based on cloud computing [18]

Shehri [19] proposed a Secure Mobile Learning Framework (SMLF) based on Trusted Platform Module (TPM) in the cloud as shown in Figure 4. The framework is supported by three layers that help in ensuring security, which are: Mobile Learning Application Layer, Protocol Layer, and Physical Infrastructure Layer. Also, the researcher introduced a procedure for personalizing mobile learning applications for learners and instructors as shown in Figure 5. The researcher claimed that the proposed framework ensures mutual authentication of all stakeholders, integrity of the message, privacy of the message, and it is free from known attacks.

Jinlai [20] introduced a live CBML framework to provide learners with the knowledge from centralized shared resources anywhere and anytime, as shown in Figure 6. To improve the availability and usability of mobile learning, cloud environment is adopted. So, the whole services are implemented at the cloud environment. In his framework, the instructor needs to register first, and then can login. When the instructor logs in, a webcam is enabled and starts to capture the session. Every live video clips is progressively downloaded to the server. Leaners who want to watch these live videos have to register and get the permission to watch them. Once the learners log in, they can view the available online instructors and select the one they want. Then, learners can access the live contents over the server. The data might be text documents, audio, or video files which are buffered to learners' mobile devices from the server. 
Sun et al. [21] designed a comprehensive cloud-based framework that builds a virtual learning environment to let both learners and instructors engage through mobile devices, as shown in Figure 7. All applications and services in the virtual learning environment will work in combination and be deployed over the cloud to use the massive storage space and high computing capability. The framework of the proposed Software as a Service (SaaS) and Micro Learning as a Service (MLaaS) is shown in Figure 8.

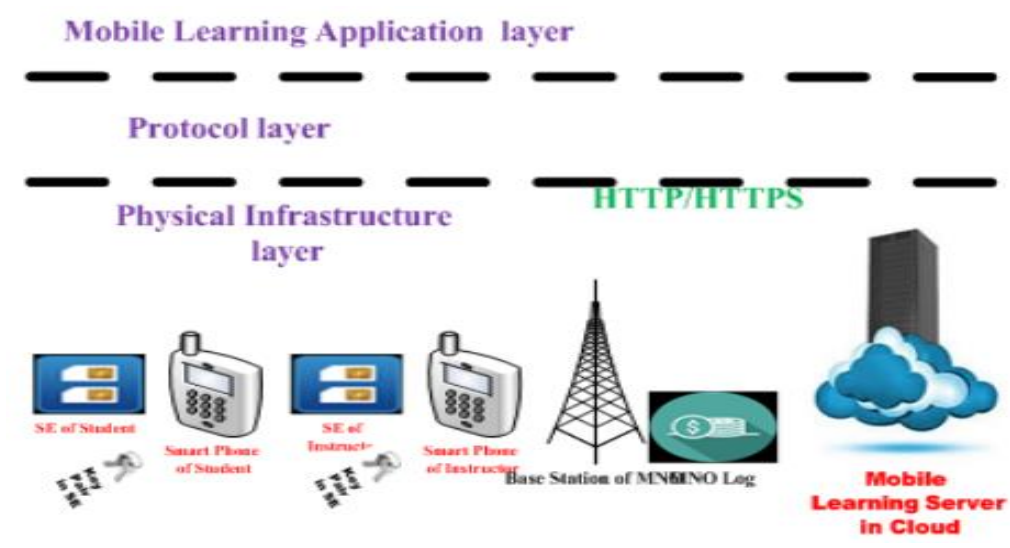

Figure 4. Secure mobile learning framework based on the cloud [19]

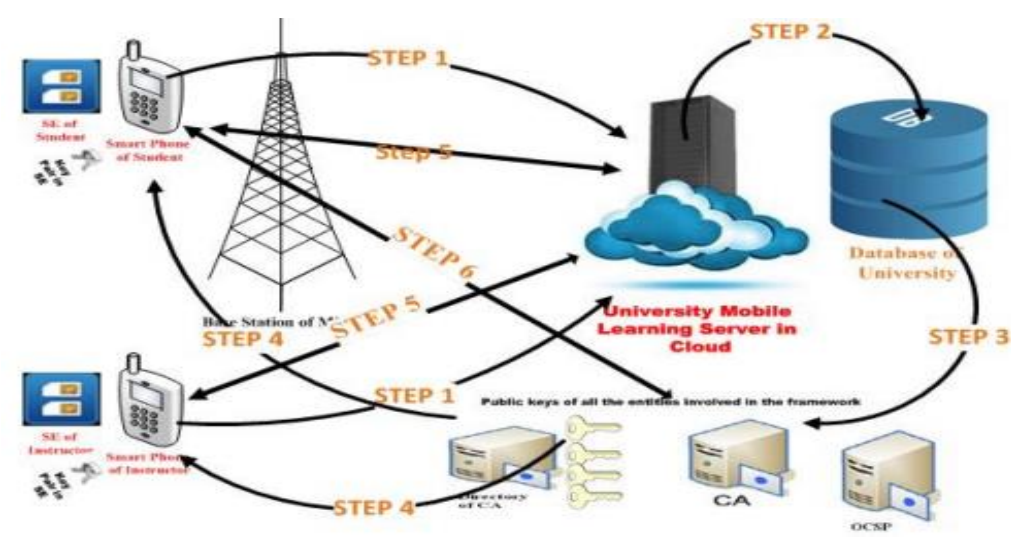

Figure 5. Procedure for personalization of secure mobile learning framework based on the cloud [19]

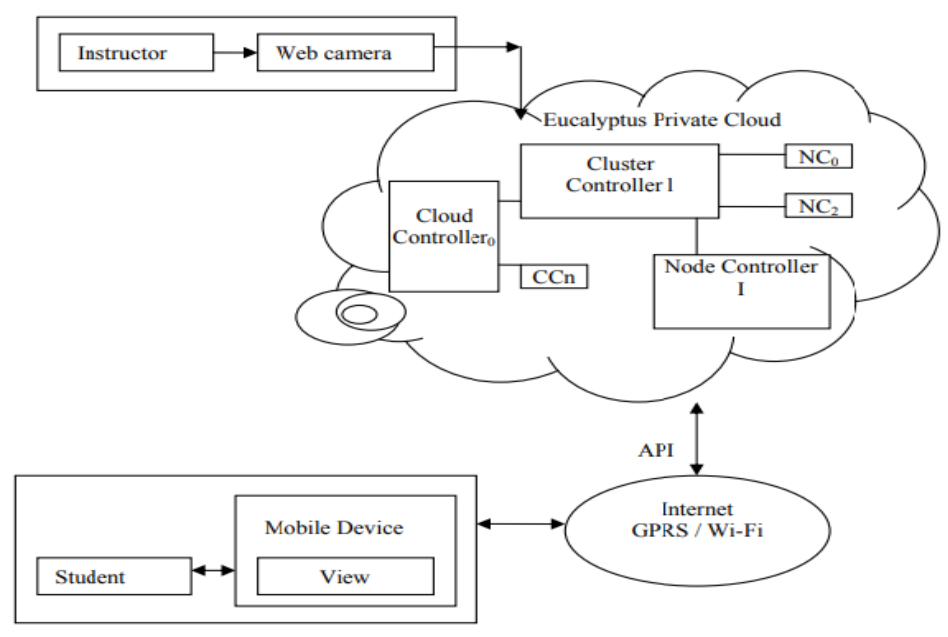

Figure 6. A live CBML framework [20] 


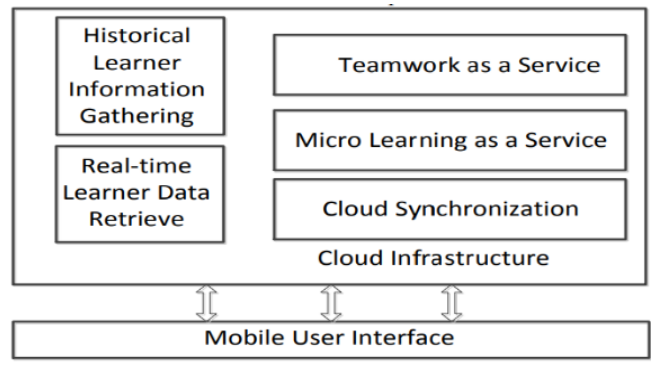

Figure 7. Framework of cloud based mobile virtual learning environment for massive open online course [21]

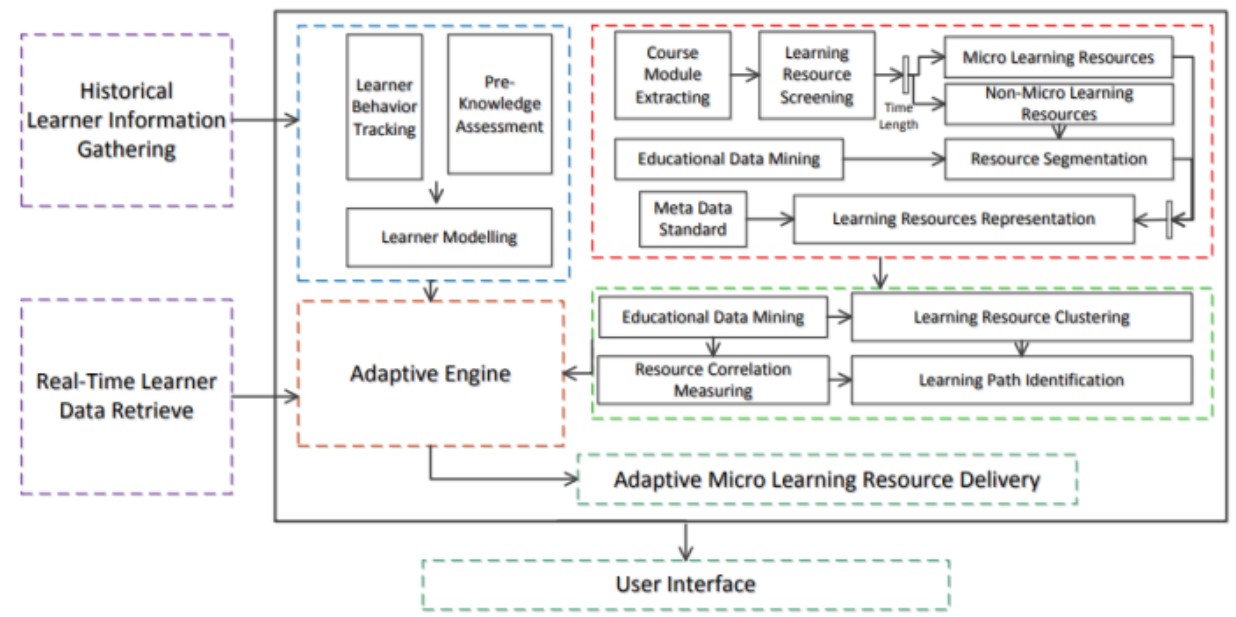

Figure 8. The Framework of the proposed saas and MLaaS [21]

\section{RESULTS AND ANALYSIS}

The use of modern technology, especially mobile devices and cloud computing in the educational sector has opened the doors for both learners and teachers for new and innovative ideas. Although there are many advantages of adopting CBML systems, there are also many limitations. The researchers have attempted to identify, explore, categorize, or evaluate the components of the CBML systems in form of frameworks. The existing CBML frameworks have some limitations that need to be addressed for mass adaptation [19] since they don't address all issues related to implementing CBML in the educational process, such as: security, usability, limited control, networks availability and downtime, evaluation challenges, device compatibility, and etc. Generally, the main hindrance in accepting mobile learning is the lack of cohesive framework [22] and this becomes more vague when integrating it with cloud computing. The inadequacy of such patterns is due to the deficiency of permanent and innovative CBML projects around the world.

The presented frameworks shared the same main themes of technical issues, learners, learning environment design, pedagogy, content, context, devices, time, usability, social interactions, system evaluation, and culture. They almost concentrated on technical issues and ignored CBML systems' outcomes, or focused on learners' acceptance or outcomes and ignored other stakeholders. Also, some frameworks are designed to a specific goal, country, university, group of users, or course.

\section{CONCLUSION}

The most important technology nowadays which may support mobile learning systems is cloud technology. The benefits of integrating mobile learning and cloud computing as a CBML system has been recognized by many educational institutions. CBML is positively affects the teaching and learning process. Learners and instructors have the opportunity to transfer rapidly and economically to CBML applications through their mobile devices. This transformation reduces the operational cost at educational institutions, the costs of software licenses, maintenance, and materials. Also, it provides more powerful functional capabilities. Furthermore, the use of new technology in the educational process has opened the doors for innovative and new thoughts and ideas to get luxury and improvement for both students' and teachers' lives. 
Many researches have attempted to syndicate the features of CBML in a form of a framework. More studies and investigations are required to fill in the gap of enhancing CBML frameworks towards more standardized and generalized with intelligence features since most of the conducted CBML frameworks were limited to a specific country, university or college, group of users, course, or specific goal. Actually, understanding mobile learning and cloud computing technologies supports designing an effective and dependable CBML framework.

\section{ACKNOWLEDGEMENTS}

The authors would like to thank Computer Science Department at the School of Informatics \& Applied Mathematics, Universiti Malaysia Terengganu (UMT), Malaysia, for their support in preparing this paper.

\section{REFERENCES}

[1] W. Jin and D. Zhirui, "Research on Mobile Learning Model of College English Based on WeChat Platform," Eurasia Journal of Mathematics, Science and Technology Education, vol. 13, pp. 5847-5853, 2017.

[2] S. Dhanalakshmi, et al., "Mobile learning using cloud computing," International Journal of Computer and Engineering (IJECE), vol. 2, pp. 102-108, 2014.

[3] S. Alamri, et al., "Learning Anytime, Anywhere: Benefits and Challenges of M-Learning through the Cloud," International Advanced Research Journal in Science, Engineering and Technology (ARJSET), vol. 4, pp. 37-41, 2017.

[4] S. Miller, et al., "Cloud-based Machine Learning for the detection of anonymous web proxies," $27^{\text {th }}$ Irish Signals and Systems Conference, 2016, pp. 1-6.

[5] O. Harfoushi, "Influence of Cloud Based Mobile Learning Applications on User Experiences: A Review Study in the Context of Jordan," International Journal of Interactive Mobile Technologies, vol. 11, pp. 202-211, 2017.

[6] G. Thejesvi and E. Hariprasad, "Mobile based Learning through the Cloud Technology," International Journal of Computer Science and Information Technologies (IJCSIT), vol. 7, pp. 2537-2540, 2016.

[7] H. He, et al., "Innovation of teaching methods in university based on mobile cloud computing," World Transactions on Engineering and Technology Education, vol. 14, pp. 208-213, 2016.

[8] O. Boyinbode and F. Akintade, "A cloud based mobile learning interface," Proceedings of the World Congress on Engineering and Computer Science, San Francisco, USA, vol. 1, 2015.

[9] U. Kamalu and U. Onyedumekwu, "Applications and Derivable Benefits of Cloud-Based Mobile Learning Platform in Nigeria Higher Institutions," International Journal of Advanced Research in Computer Science and Software Engineering, vol. 6, pp. 141-146, 2016.

[10] G. Yusufu and N. Nathan, "Cloud-Based Mobile Learning for Higher Education in Nigeria: A Review," Adamawa State University Journal of Scientific Research, vol. 4, pp. 13-22, 2016.

[11] D. Aniobi and E. Alu, "Usability Testing and Evaluation of a Cloud Computing-based Mobile Learning App: Students' Perspective," IJCSI International Journal of Computer Science Issues, vol. 13, pp. 67-75, 2016.

[12] T. Kobayashi, et al., "An Application Framework for Smart Education System Based on Mobile and Cloud Systems," IEICE TRANS. INF. \& SYST., vol. E100-D, pp. 2399-2410, 2017.

[13] N. Katiyar and R. Bhujade, "Framework to Cloud Computing Based M-Learning for Higher Education: an Adopt," International Journal of Engineering Trends and Technology, vol. 61, pp. 0-48, 2018.

[14] E. Badidi, "A Cloud-based Framework for Personalized Mobile Learning Provisioning using Learning Objects Metadata Adaptation," CSEDU 2016 Proceedings of the $8^{\text {th }}$ International Conference on Computer Supported Education, vol. 1, pp. 368-375, 2016.

[15] Z. Rimale, et al., "Survey on the Use of the Mobile Learning Based on Mobile Cloud Computing," International Journal of Interactive Mobile Technologies, vol. 10, pp. 35-41, 2016.

[16] A. Baalghusun and R. Qureshi, "A Novel Model for Cloud Based Mobile Learning System," International Journal of Information Engineering and Electronic Business, vol. 6, pp. 40-46, 2014.

[17] A. Brhanu and H. Mulugeta, "Conceptual Framework to Adopt Cloud Based M-Learning for Higher Education Institutions: Ethiopian Perspective," International Journal of Innovative Research in Science, Engineering and Technology, vol. 4, pp. 10328-10335, 2015.

[18] W. G. Alghabban, et al., "M-Learning: Effective Framework for Dyslexic Students Based on Mobile Cloud Computing Technology," International Journal of Advanced Research in Computer and Communication Engineering, vol. 5, pp. 513-517, 2016.

[19] M. Shehri, "A Secure Mobile Learning Framework based on Cloud," International Journal of Advanced Computer Science and Applications," IJACSA, vol. 8, pp. 7-11, 2017.

[20] Y. Jinlai, "The Building of Mobile Interactive Learning System based on Cloud Computing," International Journal of Digital Content Technology and its Applications (JDCTA), vol. 6, pp. 266-274, 2012.

[21] G. Sun, et al., "MLaaS: A cloud system for mobile micro learning in MOOC," IEEE International Conference on Mobile Services, United States: IEEE, pp. 120-127, 2015.

[22] M. B. Ada, "Using Design-Based Research to Develop a Mobile Learning Framework for Assessment Feedback," Research and Practice in Technology Enhanced Learning, vol. 13, pp. 1-22, 2018. 